\title{
HEMORHEOLOGICAL TESTS IN CARDIOVASCULAR DISEASES
}

(Contribution to the Round Table Discussion at the 9 th European Conference on Clinical Hemorheology, Siena June 29-July 1 1995)

Amparo Vayá

"La Fe" Hospital. Valencia. Spain.

Patients with cardiovascular risk factors or cardiovascular diseases frequently show increased blood viscosity (BV), plasma viscosity, hematocrit, erythrocyte aggregation and decreased red blood cell (RBC) deformability. It is well known that $\mathrm{BV}$ is a global test which combines the rheological effects of all the components of blood. Therefore, an alteration in any one of these determinants could be expected to significantly increase BV. The findings of different authors and our own results indicate that patients with cardiovascular risk factors and thrombotic arterial or venous diseases can have a blood viscosity value in the normal range even when some determinants are altered. BV cannot by itself be considered an overall screening test. It only reflects the behavior of the blood in large vessels. Plasma viscosity and erythrocyte deformability and aggregation must also be measured for they play an important role in the development of ischemic events at the microcirculatory level.

With respect to individuals factors, any given rheological test (RBC deformability) can, in a single pathology e.g. hyperlipoproteinemia yield contradictory results depending on the measurement system employed. Since a decrease in RBC deformability can be due to various causes (cell geometry, internal viscosity and membrane viscoelasticity), the equipment used must be sensitive and specific enough to detect alterations in these determining factors. A decreased RBC deformability can be improved as long as the cause is identified and the appropriate drug is administered.

With respect to the other relevant $\mathrm{RBC}$ rheological property (RBC aggregation) which determines blood flow at low shear, many authors suggest that it mainly depends on the fibrinogen level and that it need not be measured for the two parameters are strongly correlated. In our own experience in deep vein thrombosis patients and in patients with cardiovascular risk factors, the fibrinogen measurement can not serve as a substitute for assessment of erythrocyte aggregation because other plasmatic factors and membrane structure modifications are involved in the formation of red blood cell aggregates. 
Round Table Discussion at the 9th European Conference on Clinical Hemorheology, Siena June 29-July 11995

\section{PLENARY DISCUSSION}

H. Schmid-Schönbein responded to the contribution of Rampling by saying that hemodilution does have beneficial effects, at least in the field of ophthalmology. D. Lerche commented on Forconi's presentation with respect to the need for a golden standard in the measurement of whole blood viscosity. He stated that the Fahraeus-Lindquist effect is indeed present during rotational viscosimetry of blood at low hematocrit.

Schmid--Schönbein recalled a previous publication from his hand about a method to plot the laboratory results of hemorheological parameters in a way that is instrument independant, the so-called Aachen Test Profile (*).

Ehrly, reacting on Hardeman's Introductory remarks, emphasized that clinicians simply want to have a number in stead of a curve e.g. for whole blood viscosity (see also under Introductory Remarks, possible approaches A). Furthermore, he stated that we should not bother about the accuracy of a certain measurement as long as the clinical relevance of that parameter is not known. Hardeman emphasised that differences in clinical hemorheological parameters are usually very small and in order to prove their clinical significance, a high accuracy of their measurement is a prerequisite.

Finally, Di Perri refered to some recent ,not yet published, work of his group about the significant local variations in viscosity and shear rate measured during systole and diastole at the carotid bifurcation along the proximal and distal vessel walls and in the internal and external carotid artery on both the left and right side of 54 normal human subjects.

* SCHMID-SCHöNBEIN, H., TEITEL, P. The Aachen Clinical Hemorheology Test Profile: proposal for the documentation of hemorheological data in clinical medicine. Biorheology, suppl. 1, 49-62, 1984 
Round Table Discussion at the 9th European Conference on Clinical

Hemorheology, Siena June 29-July 11995

\section{CONCLUSIVE REMARKS}

\section{M.R.Hardeman}

The following recommendations can be abstracted from this Round Table Discussion:

1. In clinical studies we should determine all possible determinants of blood viscosity, however, we should be very much aware of instrumental and technical differences. Standardisation is of the essence; a lot of work to do for the Expert Panels!

2. In order to increase the acceptance of laboratory results by clinicians the results should be expressed as much as possible by a single number.

3. Emphasis has to be placed on blood as an organ i.e. inclusive the endothelium. Furthermore, the pulsative character of blood flow should be considered in laboratory determinations to a greater extend than is currently done.

4. The assessment of the effect of drugs on hemorheological parameters should preferably performed ex vivo and in combination with the patient's clinical progress.

5. Local differences of viscosity determining factors, in relation to those prevailing in "systemic" blood should be recognised and corrected for as much as possible.

6. Although it still does not appear possible to directly extrapolate data and information obtained from in vitro (ex vivo) studies to the in vivo situation and these data can only be seen as a means to assess a "factor of risk" (*), we can state that, with these restrictions and conditions in mind, hemorheological laboratory parameters might have the potency to be clinically relevant.

* TEITEL, P. The contribution of haemorheology to the understanding of microcirculatory disorders. An introductory overview. Drugs 33 (suppl. 2), 5-11, 1987. 